\title{
Research Note \\ Dust Alfvén envelope solitons in astrophysical dusty plasmas
}

\author{
P. K. Shukla ${ }^{1, \star}$ and F. Verheest ${ }^{2}$ \\ 1 Institut für Theoretische Physik IV, Ruhr-Universität Bochum, 44780 Bochum, Germany \\ 2 Sterrenkundig Observatorium, Universiteit Gent, Krijgslaan 281, 9000 Gent, Belgium
}

Received 5 December 2002 / Accepted 13 February 2003

\begin{abstract}
It is shown that nonlinear dynamics of modulated dust Alfvén wave packets is governed by a derivative nonlinear Schrödinger equation. The latter admits localized envelope solitons whose width $L$ is larger than $V_{\mathrm{A}} / \Omega_{\mathrm{d}}$, where $V_{\mathrm{A}}$ is the dust Alfvén speed and $\Omega_{\mathrm{d}}$ the dust gyrofrequency. For dusty plasma parameters relevant to molecular clouds in interstellar space, the soliton width is of the order of a fraction of a parsec.
\end{abstract}

Key words. plasmas - waves - ISM: clouds

Charged dust is ubiquitous in astrophysical plasmas such as those in cometary tails, interstellar molecular clouds, and planetary nebulae (see e.g. Nakano 1984; Bliokh et al. 1995; Hartquist et al. 1997; Kamaya \& Nishi 1998; Zweibel 1999; Kamaya \& Nishi 2000; Nakano et al. 2002; Mendis 2002). In magnetized astrophysical plasmas, charged dust grains are responsible for new wave modes, including obliquely (to the magnetic field direction) propagating dust-acoustic waves and magnetic-field aligned dust Alfvén waves (Mendis \& Rosenberg 1994; Shukla 1999; Shukla \& Mamun 2002), whose frequencies are much smaller than the ion gyrofrequency. In a nonuniform dusty magnetoplasma, the magnetic fields are decoupled from the plasma due to the violation of the frozen-infield condition (Birk et al. 1996; Rudakov 2001). Here, one can have a possibility of nonlinear structures including shocks and vortices (Rudakov 2001; Shukla \& Mamun 2001).

In this paper we consider the nonlinear propagation of magnetic field-aligned dispersive dust Alfvén (DDA) waves (Shukla \& Mamun 2002) and show that the DA wave dynamics is governed by a derivative nonlinear Schrödinger (DNLS) equation. The latter admits localized envelope solitons, which can account for large scale structures in interstellar media containing small scale irregularities.

Let us consider right-hand circularly polarized (RHCP) DDA waves in a magnetized plasma composed of electrons, ions, and negatively charged dust grains. At equilibrium we have

$n_{\mathrm{i} 0}=n_{\mathrm{e} 0}+Z_{\mathrm{d}} n_{\mathrm{d} 0}$,

where $n_{j 0}$ is the equilibrium density of particle species $j$ ( $j$ equals "e" for the electrons, "i" for the ions, and " $\mathrm{d}$ " for dust

Send offprint requests to: P. K. Shukla,

e-mail: ps@tp4.ruhr-uni-bochum.de

* Also at the Department of Plasma Physics, Umeå University, 90187 Umeå, Sweden. grains), and $Z_{\mathrm{d}}$ the number of charges residing on the dust grain surface. We consider low-frequency DDA waves (in comparison with the ion gyrofrequency $\Omega_{\mathrm{i}}=e B_{0} / m_{\mathrm{i}}$ ), where $e$ is the magnitude of the electron charge, $B_{0}$ the strength of the ambient (unperturbed) magnetic field, and $m_{\mathrm{i}}$ the ion mass. The dispersion relation of such low-frequency DDA waves parallel to $\widehat{z}$ is

$\frac{k^{2} c^{2}}{\omega^{2}} \simeq \frac{\omega_{\mathrm{pe}}^{2}}{\omega \Omega_{\mathrm{e}}}-\frac{\omega_{\mathrm{pi}}^{2}}{\omega \Omega_{\mathrm{i}}}-\frac{\omega_{\mathrm{pd}}^{2}}{\omega\left(\omega-\Omega_{\mathrm{d}}\right)}$,

where $\widehat{z}$ is the unit vector along the $z$ axis, $\omega$ and $k$ are the frequency and wave number, respectively, $\omega_{\mathrm{p} j}=\left(n_{j 0} q_{j}^{2} / \varepsilon_{0} m_{j}\right)^{1 / 2}$ is the plasma frequency, $\Omega_{j}=\left|q_{j}\right| B_{0} / m_{j}$ the gyrofrequency, $m_{j}$ the mass, $q_{\mathrm{e}}=-e, q_{\mathrm{i}}=e$ and $q_{\mathrm{d}}=-Z_{\mathrm{d}} e$. The cold plasma dispersion relation (2) is valid for $\omega \ll c k$ and $k V_{T j} \ll\left|\omega-\Omega_{j}\right|$, where $V_{T j}=\left(\kappa_{\mathrm{B}} T_{j} / m_{j}\right)^{1 / 2}$ is the thermal speed, $\kappa_{\mathrm{B}}$ is the Boltzmann constant, and $T_{j}$ the temperature. We have also assumed that $v_{\mathrm{en}} \ll \Omega_{\mathrm{e}}$ and $v_{\text {in }} \ll \Omega_{\mathrm{i}}$, so that collisions of electrons and ions with neutrals are insignificant. Here $v_{\mathrm{en}}$ and $v_{\text {in }}$ are the electron-neutral and ion-neutral collision frequencies, respectively. Dust-neutral collisions can be ignored if the DDA wave frequency is larger than the dustneutral collision frequency $v_{\mathrm{dn}}$. We note that in collisionless dusty magnetoplasmas the first two terms, which represent the contributions of the $\boldsymbol{E}_{\perp} \times \boldsymbol{B}_{0}$ currents, in the right-hand side of (2) yield $-\omega_{\text {pd }}^{2} / \omega \Omega_{\mathrm{d}}$, where $\boldsymbol{E}_{\perp}$ is the perpendicular (to $\widehat{\boldsymbol{z}}$ ) component of the DDA wave electric field. Hence, (2) reduces to

$\frac{k^{2} c^{2}}{\omega^{2}} \simeq \frac{\omega_{\mathrm{pd}}^{2}}{\Omega_{\mathrm{d}}\left(\Omega_{\mathrm{d}}-\omega\right)}$,

which gives for $\omega \ll \Omega_{\mathrm{d}}$ the DDA wave frequency

$\omega \simeq k V_{\mathrm{A}}\left(1-\frac{k V_{\mathrm{A}}}{2 \Omega_{\mathrm{d}}}\right)$ 
Here $V_{\mathrm{A}}=B_{0} /\left(\mu_{0} n_{\mathrm{d} 0} m_{\mathrm{d}}\right)^{1 / 2}$ is the dust Alfvén speed. Equation (4) shows that dust Alfvén waves are weakly dispersive.

Finite amplitude DDA waves interacting nonlinearly with background density fluctuations produce envelope of DDA waves, which are governed by the DNLS equation (Verheest 2000)

$$
\frac{\partial}{\partial \tau} \boldsymbol{B}_{\perp}+\frac{V_{\mathrm{A}}}{4 B_{0}^{2}} \frac{\partial}{\partial \zeta}\left(\left|B_{\perp}\right|^{2} \boldsymbol{B}_{\perp}\right)-\frac{V_{\mathrm{A}}^{2}}{2 \Omega_{\mathrm{d}}} \widehat{z} \times \frac{\partial^{2}}{\partial \zeta^{2}} \boldsymbol{B}_{\perp}=0
$$

where $\boldsymbol{B}_{\perp}$ is the perpendicular component of the wave magnetic field, $\tau$ and $\zeta=z-V_{\mathrm{A}} t$ are the slowly varying time and space coordinates. We note that Eq. (5) can be readily derived from the dusty plasma magnetohydrodynamic equations (Shukla \& Mamun 2002) by using a multiple-time-and-space-scale analysis (Verheest 2000).

Introducing $\psi=\left(B_{x}+i B_{y}\right) / B_{0}$ for circular polarization, we obtain from (5)

$$
\frac{\partial \psi}{\partial \tau}+\frac{V_{\mathrm{A}}}{4} \frac{\partial}{\partial \zeta}\left(|\psi|^{2} \psi\right)-i \frac{V_{\mathrm{A}}^{2}}{2 \Omega_{\mathrm{d}}} \frac{\partial^{2} \psi}{\partial \zeta^{2}}=0
$$

Equation (6) admits localized envelope soliton solutions

$|\psi|^{2}=\left|B_{\perp}\right|^{2}=\frac{8 V}{V_{\mathrm{A}}}\left[\sqrt{2} \cosh \left(\frac{\zeta-V \tau}{L}\right)-1\right]^{-1}$,

where $V$ is a constant and $L=V_{\mathrm{A}}^{2} / 2 V \Omega_{\mathrm{d}} \gtrsim 5 V_{\mathrm{A}} / \Omega_{\mathrm{d}}$ is the width of the soliton and $8 V / V_{\mathrm{A}} \lesssim 1$ is a measure for the maximum amplitude of the soliton. We take typical plasma parameters in interstellar molecular clouds, namely $n_{\mathrm{d} 0}=10^{-1} \mathrm{~m}^{-3}$, $Z_{\mathrm{d}}=10, B_{0}=10 \mu \mathrm{G}=1 \mathrm{nT}, m_{\mathrm{d}} \simeq 10^{12} m_{\mathrm{i}}$ and obtain $V_{\mathrm{A}}=100 \mathrm{~m} \mathrm{~s}^{-1}, \Omega_{\mathrm{d}} \simeq 10^{-12} \mathrm{~s}^{-1}$, and hence $L \gtrsim 5 \times 10^{14} \mathrm{~m} \simeq$ $0.02 \mathrm{pc}$. We note that the amplitude of the envelope DDA solitons would be slightly reduced when the dust-neutral collisions are taken into consideration, as one (Rudakov 2001) typically has $v_{\mathrm{dn}} / \Omega_{\mathrm{d}} \ll 1$. Furthermore, consideration of a nonambipolar diffusion due to interparticle collisions may give rise to envelope shock-like structures due to the dissipation of the DDA wave magnetic field energy.

The length scale $L$ thus obtained is large compared to the Jeans length $\lambda_{\mathrm{Jd}}$ corresponding to the charged dust component, since the dust Jeans frequency for the parameters quoted is $\omega_{\mathrm{Jd}} \simeq 3 \times 10^{-13} \mathrm{~s}^{-1}$, and at an average dust temperature of $T_{\mathrm{d}}=30 \mathrm{~K}$ the dust thermal velocity is $v_{\mathrm{Td}} \simeq 5 \times 10^{-4} \mathrm{~m} \mathrm{~s}^{-1}$. This results in a dust Jeans length of $\lambda_{\mathrm{Jd}} \simeq 10^{10} \mathrm{~m} \ll L$.
Even if the dust-acoustic velocity $c_{\mathrm{da}}$ is used to compute the dust Jeans length, that increases $\lambda_{\mathrm{Jd}}$ with a factor $10^{2}$, so that the same conclusion remains valid (Verheest \& Čadež 2002). All these estimates have to be taken as indications, of course, because of the uncertainties about the parameters of charged dust component.

To summarize, we have considered the nonlinear propagation of magnetic field-aligned dispersive dust Alfvén waves in magnetized dusty plasmas. It is shown that the self-modulation of DDA waves occurs due to their nonlinear interaction with the ambient dusty plasma. The dynamics of modulated (by zero-frequency density fluctuations) DDA waves is governed by a derivative nonlinear Schrödinger equation, which admits envelope DDA solitons. For the plasma parameters in interstellar molecular clouds, the scale size of the DDA wave envelope solitons is a fraction of a pc. Hence, these nonlinear waves can account for large scale structures in astrophysical environments.

Acknowledgements. The work of PKS was supported by the Deutsche Forschungsgemeinschaft (Bonn) through the Sonderforschungsbereich 591. FV thanks the Fonds voor Wetenschappelijk Onderzoek (Vlaanderen) for a research grant.

\section{References}

Birk, G. T., Kopp, A., \& Shukla, P. K. 1996, Phys. Plasmas, 3, 3564

Bliokh, P., Sinitsin, V., \& Yaroshenko, V. 1995, Dusty and SelfGravitational Plasmas in Space (Dordrecht: Kluwer)

Hartquist, T. W., Pilipp, W., \& Havnes, O. 1997, Astrophys. Space Sci., 246, 243

Kamaya, H., \& Nishi, R. 1998, ApJ, 500, 257

Kamaya, H., \& Nishi, R. 2000, ApJ, 543, 257

Mendis, D. A., \& Rosenberg, M. 1994, ARA\&A, 32, 419

Mendis, D. A. 2002, Plasma Sources Sci. Technol., 11, A219

Nakano, T. 1984, Fund. Cosmic Phys., 9, 139

Nakano, T., Nishi, R., \& Umebayashi, T. 2002, ApJ, 573, 199

Rudakov, L. I. 2001, Phys. Scr., T89, 158

Shukla, P. K. 1992, Phys. Scr., 45, 504

Shukla, P. K. 1999, Phys. Lett. A, 252, 340

Shukla, P. K., \& Mamun, A. A. 2001, Phys. Scr., 64, 351

Shukla, P. K., \& Mamun, A. A. 2002, Introduction to Dusty Plasma Physics (Bristol: IOP)

Verheest, F. 2000, Waves in Dusty Space Plasmas (Dordrecht: Kluwer) Verheest, F., \& Čadež, V. M. 2002, Phys. Rev. E, 66, 056404

Zweibel, E. G. 1999, Phys. Plasmas, 6, 1725 